\title{
Cellular apoptosis of HFF cells by inorganic nanoparticles not susceptible to modulation by Toxoplasma gondii infection in vitro
}

\author{
Oluyomi Stephen Adeyemi ${ }^{\mathrm{a}, *}$, David Adeiza Otohinoyi ${ }^{\mathrm{b}}$, Oluwakemi Josephine Awakan ${ }^{\mathrm{a}}$, \\ Adebukola Anne Adeyanju ${ }^{c}$ \\ a Medicinal Biochemistry, Nanomedicine \& Toxicology Laboratory, Department of Biochemistry, Landmark University, PMB 10017 Omu-Aran, Nigeria \\ ${ }^{\mathrm{b}}$ School of Medicine, All Saints University, Hillsborough Street, Roseau, Dominica \\ ${ }^{\mathrm{c}}$ Department of Biological Sciences, Mcpherson University, Seriki-Sotayo, Ogun State, Nigeria
}

\section{A R T I C L E I N F O}

\section{Keywords:}

Cellular interaction

Infection

Metabolic modulators

Nanomedicine

\begin{abstract}
A B S T R A C T
The interaction of nanoparticles with living cells is becoming one of the urgent areas of collaborative research in materials science and biology. Previously, we showed that nanoparticles have promising anti-Toxoplasma gondii properties. Meanwhile, Toxoplasma gondii has been shown to avert apoptosis in host cells whereas nanoparticles have been implicated for apoptotic tendency. Therefore, in the present study, we assessed the in vitro apoptotic properties of inorganic nanoparticles in the absence or presence of Toxoplasma infection and/or small molecules used as metabolic modulators. Results showed that inorganic nanoparticles dose-dependently caused cellular apoptosis. However, in the presence of infection by Toxoplasma gondii, nanoparticles-induced cellular apoptosis was not mitigated. Likewise, use of several small molecules (anti-metabolites) as metabolic modulators either mildly or nearly failed to abate cellular apoptosis by nanoparticles. Taken together, our findings do not only confirm the apoptotic potential of inorganic nanoparticles but show evidence that cellular apoptosis by inorganic nanoparticles of gold and silver might not be susceptible to modulation by Toxoplasma gondii infection. The findings are new and contribute to deepen our understanding of the cellular interaction of nanoparticles.
\end{abstract}

\section{Introduction}

One of the relevant and recent advances in material science is the increasing application of nanoparticles (NPs) to transform diverse fields of biomedical science (Adeyemi et al., 2017a). For example, over the past two decades, NPs have been gaining popularity for their usage in drug delivery, therapeutics, diagnostics among others (Adeyemi and Sulaiman, 2015). Among the inorganic NPs with biological significance are silver nanoparticles (AgNPs), gold nanoparticles (AuNPs) and platinum nanoparticles (PtNPs). AgNPs have been implicated for cytotoxic efficacy applicable in cancer therapy (Azizi et al., 2017). AuNPs are inert and biocompatible and these have been applied as theranostics as well as for phototherapy (Moses et al., 2016; Rieznichenko et al., 2012) while PtNPs are noted for antioxidant properties that mimic catalase and superoxide dismutase (Sulaiman et al., 2015; Adeyemi et al., 2018a). As a further testament of the increasing popularity of NPs for biomedical applications, the potential of NPs against Toxoplasma gondii infection was recently reported (Adeyemi et al., 2017a; Adeyemi et al., 2017b). In these studies, AgNPs, AuNPs, and PtNPs, exhibited antiparasitic potential which in part was mediated through modulation of host cellular processes such as the downregulation of hypoxia inducing factor 1 protein (HIF-1 $\alpha$ ) and activation of kynurenine pathway. Interestingly, NPs and T. gondii affect similar cellular targets such as hypoxia inducing factor 1 (HIF-1 $\alpha$ ) and tryptophan pathway, particularly indoleamine 2,3-dioxygenase (IDO; rate limiting enzyme in the degradation of tryptophan to form kynurenine) (Blader and Saeji, 2009; Adeyemi et al., 2017b; Wiley et al., 2010; Spear et al., 2006; Zinkernagel et al., 2007).

Perhaps, there is a growing need to further our understanding of cellular interactions by NPs. These investigations are warranted if we consider some emerging facts; for example, the anti-T. gondii potential of inorganic NPs of silver, gold and platinum has been linked capacity to modulate cellular processes such as the metabolism of tryptophan as well as hypoxia in human foreskin fibroblast (HFF) cells (Adeyemi et al., 2017a, 2017b). Additionally, investigations have shown that NPs including silver and gold nanoparticles are capable of causing apoptosis in a variety of cell types (Selim and Hendi, 2012; Satapathy et al., 2013). On the other hand, T. gondii infection has capacity to modulate several host cellular processes in order to aid unhindered growth and replication (Blader and Saeji, 2009; Zinkernagel et al., 2007). In

\footnotetext{
* Corresponding author.

E-mail address: adeyemi.oluyomi@lmu.edu.ng (O.S. Adeyemi).
} 

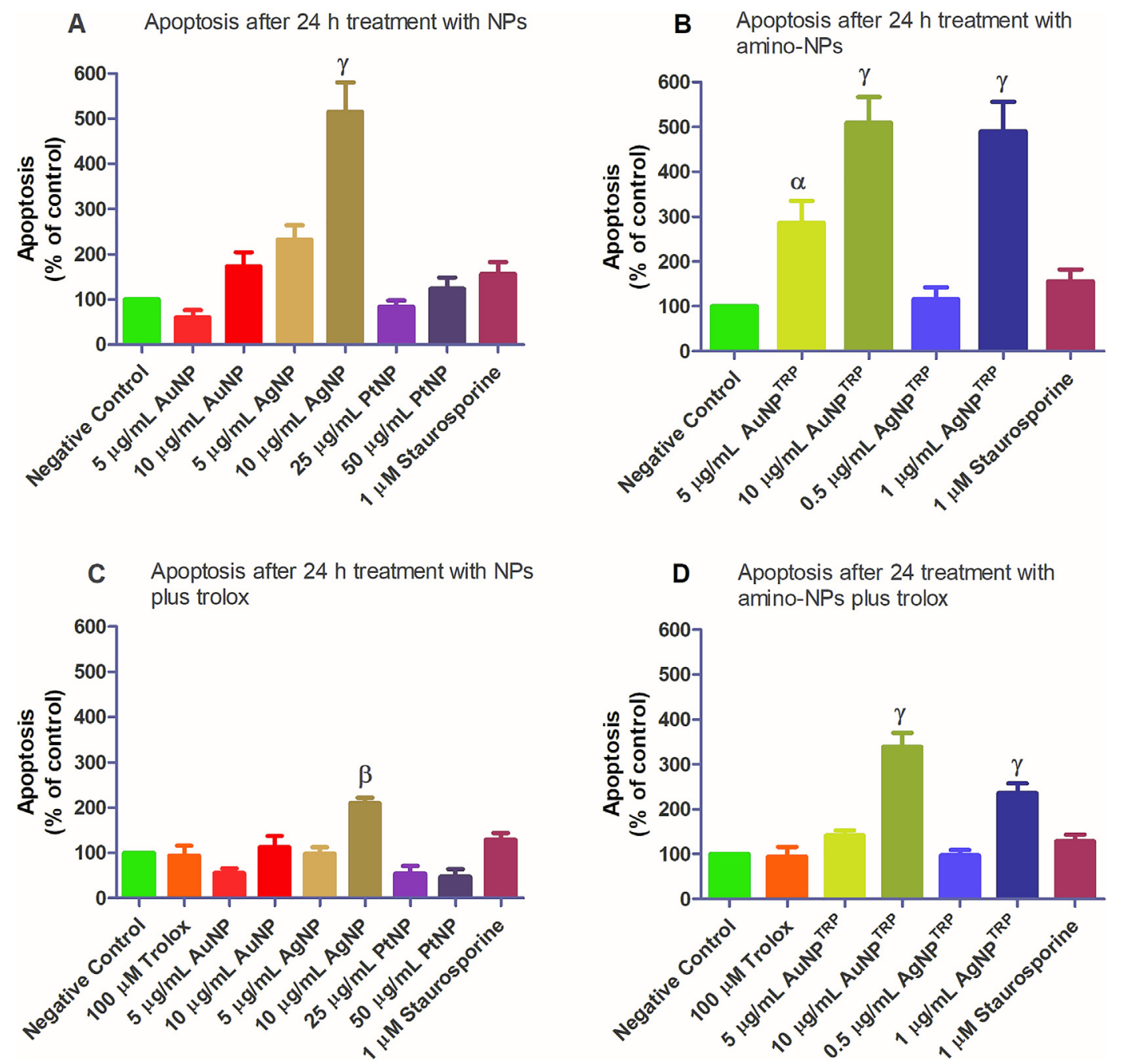

Fig. 1. Cellular apoptosis after $24 \mathrm{~h}$ treatment; [A] Nanoparticles (NPs) [B] Amino-Nanoparticles (amino-NPs) [C] Nanoparticles (NPs) plus Trolox [D] AminoNanoparticles (amino-NPs) plus Trolox. Data are presented as mean of triplicates \pm standard error of mean (SEM). The experiment was performed in triplicate and repeated three times independently. $\alpha$ is significant at $p<.05, \beta$ at $p<.001$ and $\gamma$ at $p<0.0001$ versus negative control.

A Apoptosis after treatment with NPs

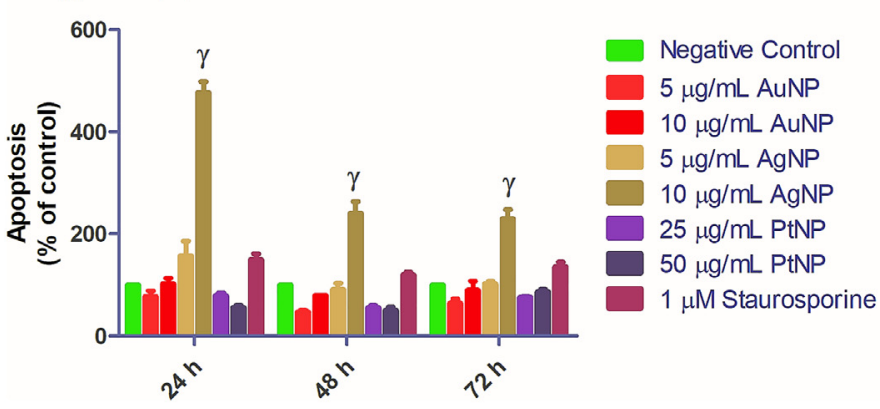

B Apoptosis after treatment with amino-NPs

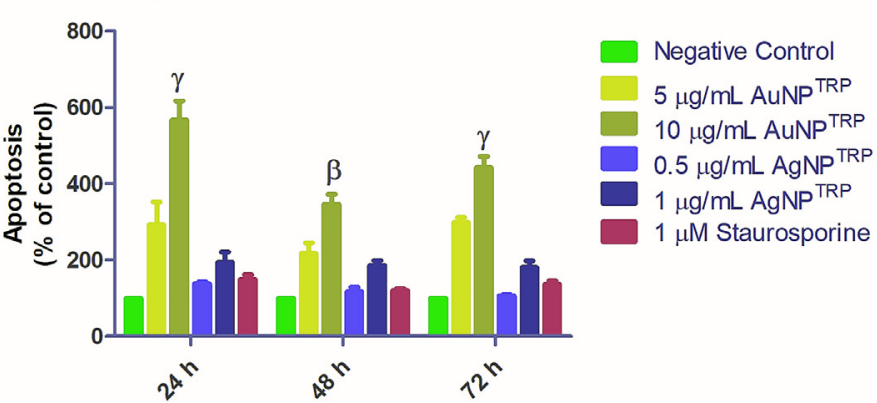

Fig. 2. Cellular apoptosis with time kinetics; [A] Treatment with nanoparticles (NPs) [B] Treatment with amino-nanoparticles (amino-NPs). Data are presented as mean of triplicates \pm standard error of mean (SEM). The experiment was performed in triplicate and repeated three times independently. $\beta$ is significant at $p<.001$ and $\gamma$ at $p<0.0001$ versus negative control.

particular, T. gondii infection can protect different cell types from apoptosis induced by a variety of pro-apoptotic treatments (Lüder and Gross, 2005; Goebel et al., 2001). Therefore, in the present study we sought to determine how the apoptotic property of NPs are affected in the presence of $T$. gondii infection and other metabolic modulators such as L-tryptophan, cobalt (II) chloride $\left(\mathrm{CoCl}_{2}\right)$, 1-Methyl-D-tryptophan (DMT), 3-(5'-Hydroxymethyl-2'-furyl)-1-benzyl indazole (YC-1), trolox (6-hydroxy-2,5,7,8-tetramethylchroman-2-carboxylic acid) and $( \pm)$
3,4-dihydro-3-hydroxy-2,2-dimethyl-4-[(phenylmethyl)amino]-2Hnaphtho[2,3-b]pyran-5,10-dione (a naphthoquinone derivative - NPQ). All of these metabolic modulators have been demonstrated to either enhance or restrict the growth of $T$. gondii elsewhere (Adeyemi et al., 2017b). 

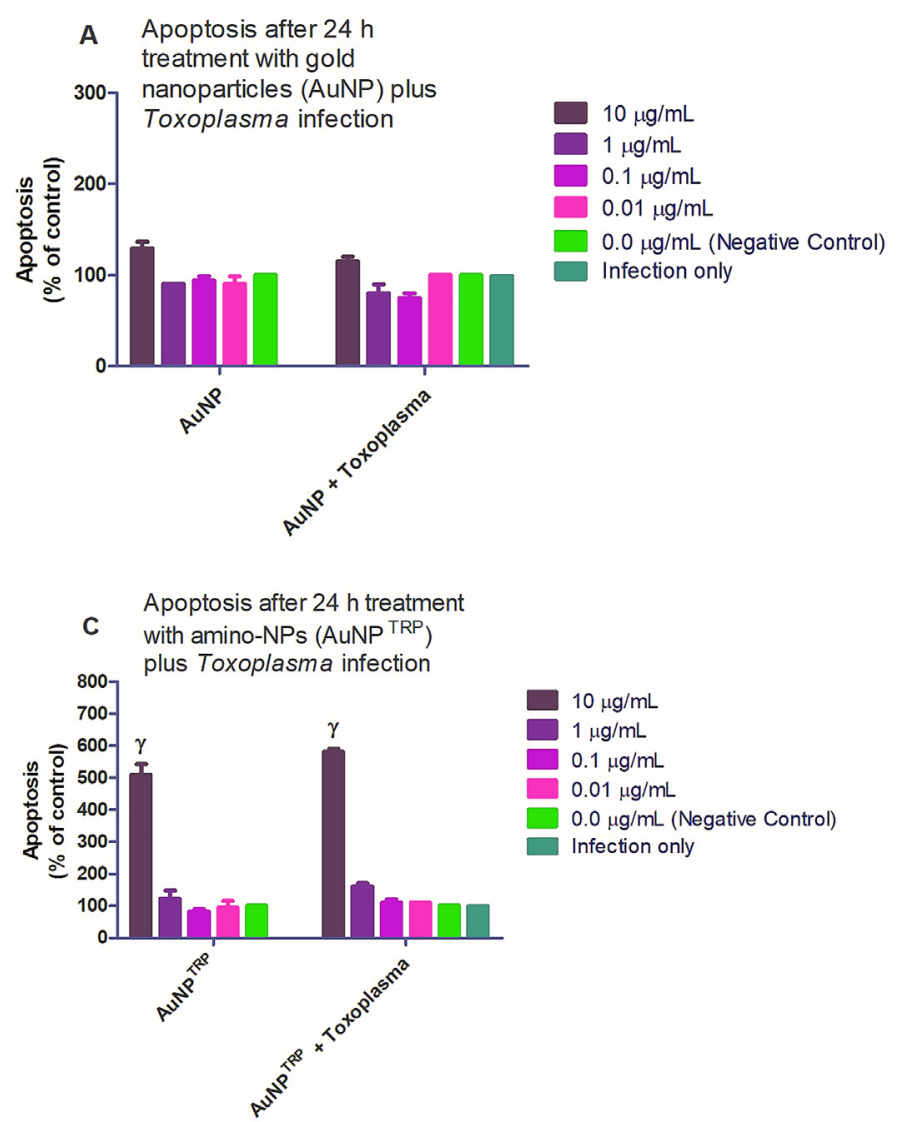
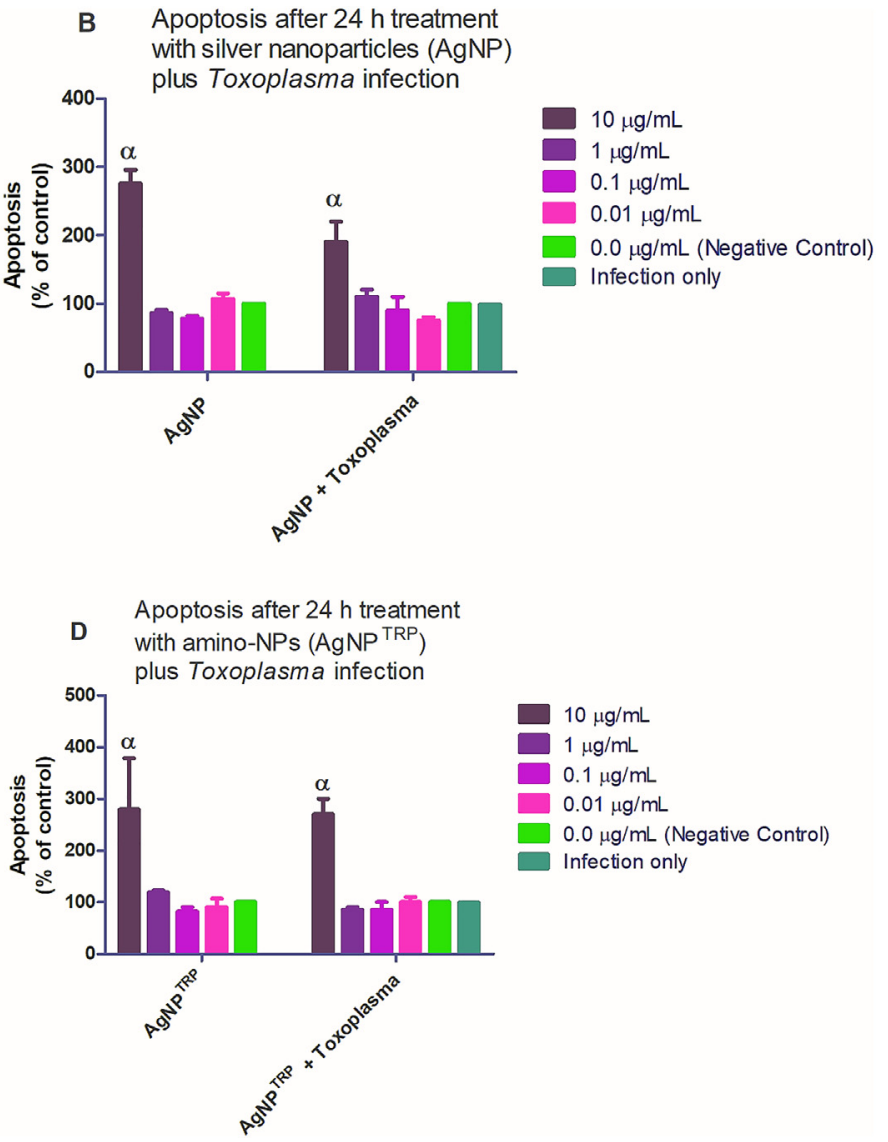

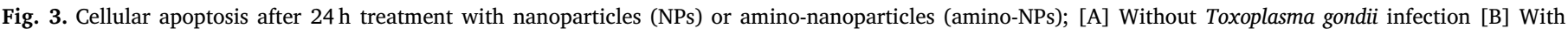

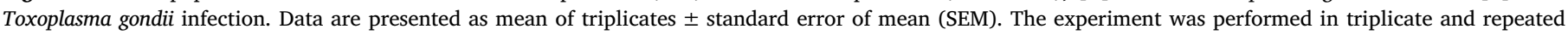
three times independently. $\alpha$ is significant at $p<.05$ and $\gamma$ at $p<0.0001$ versus negative control and/or infection only.

\section{Materials and methods}

\subsection{Materials and chemical reagents}

Nanoparticles (NPs) with diameter size range of 5-40 nm, including silver (AgNP), gold (AuNP), platinum (PtNP) and amino-NPs (silvertryptophan - AgNP ${ }^{\mathrm{TRP}}$ and gold-tryptophan - AuNP ${ }^{\mathrm{TRP}}$ ) were gifted by Global Infection Unit, NRCPD, Obihiro University of Agriculture \& Veterinary Medicine, Obihiro, Japan. Their synthesis and characterization have been reported elsewhere (Adeyemi et al., 2018b). The NPs were reconstituted in fresh culture medium prior to each use. L-tryptophan, L-kynurenine, cobalt (II) chloride $\left(\mathrm{CoCl}_{2}\right)$, 4-(dimethylamino) benzaldehyde, 1-Methyl-D-tryptophan (DMT), and 3-(5'-Hydroxymethyl-2'-furyl)-1-benzyl indazole (YC-1) were obtained from SigmaAldrich. Trolox (6-hydroxy-2,5,7,8-tetramethylchroman-2-carboxylic acid) was a product of Wako Pure Chemicals (Osaka, Japan); ( \pm )3,4dihydro-3-hydroxy-2,2-dimethyl-4-[(phenylmethyl)amino]-2H-

naphtho[2,3-b]pyran-5,10-dione (a naphthoquinone derivative - NPQ) was obtained from Cayman Chemicals (Ann Arbor, MI, USA). All other reagents were of analytical grade and used as supplied except stated otherwise.

\subsection{Anti-metabolites}

L-tryptophan is an amino acid required for T. gondii growth. $\mathrm{CoCl}_{2}$ is a chemical inducer of hypoxia while YC-1 is a potent inhibitor of hypoxia inducing factor-1 alpha (HIF-1 $\alpha$ ). Trolox is antioxidant while ( \pm )3,4-dihydro-3-hydroxy-2,2-dimethyl-4-[(phenylmethyl)amino]-

2H-naphtho[2,3-b]pyran-5,10-dione and 1-Methyl-D-tryptophan are respectively competitive and non-competitive inhibitors of indoleamine

\section{2,3-dioxygenase (IDO) activity.}

\subsection{Host cells and parasite strain}

Human fibroblast foreskin (HFF; ATCC ${ }^{\circledR}$, Manassas, VA, USA) cells were maintained at $37^{\circ} \mathrm{C}$ and $5 \% \mathrm{CO}_{2}$ atmosphere in culture medium consisting of Dulbecco's Modified Eagle's Medium (DMEM; Nissui, Tokyo, Japan), GlutaMAX ${ }^{\mathrm{TM}}-\mathrm{I}$ (Gibco, Invitrogen, UK), 10\% $(v / \mathrm{v})$ fetal calf serum (FCS; Gibco, Invitrogen, UK), penicillin and streptomycin (100 U/mL; Biowhittaker, UK) were maintained. At confluence, cells were harvested as per sub-culture protocols and resuspended to desired cell density.

The luciferase-reporting T. gondii RH strain $2 \mathrm{~F}$ (ATCC ${ }^{\circledR} 50,839$ ), expressing $\beta$-galactosidase, was used for this study. The parasite was maintained by repeated passages in human foreskin fibroblast (HFF; ATCC $^{\circledast}$ SCRC-1041) cells cultured in DMEM supplemented with GlutaMAX ${ }^{\mathrm{rm}}-\mathrm{I}, 10 \%(v / \mathrm{v})$ FCS; and penicillin and streptomycin. In order to obtain parasite suspension, host cells infected with $T$. gondii tachyzoites were passed through a 27-guage needle to lyse them and the cell lysates were then filtered through a $5-\mu \mathrm{m}$ filter to obtain a tachyzoite suspension free of host cell debris. The suspension was washed with fresh culture medium. Parasite density was determined with a hemocytometer and adjusted for in vitro experimental infection analysis at a multiplicity of infection (MOI) of 0.5 .

\subsection{Apoptosis assay}

Cellular apoptosis was detected and measured with Apoptosis Assay Cell-APOPercentage ${ }^{\mathrm{TM}}$ reagent (Biocolor Life Science Assays, Antrim, UK) by following the manufacturer's protocol. Briefly, growing HFF 

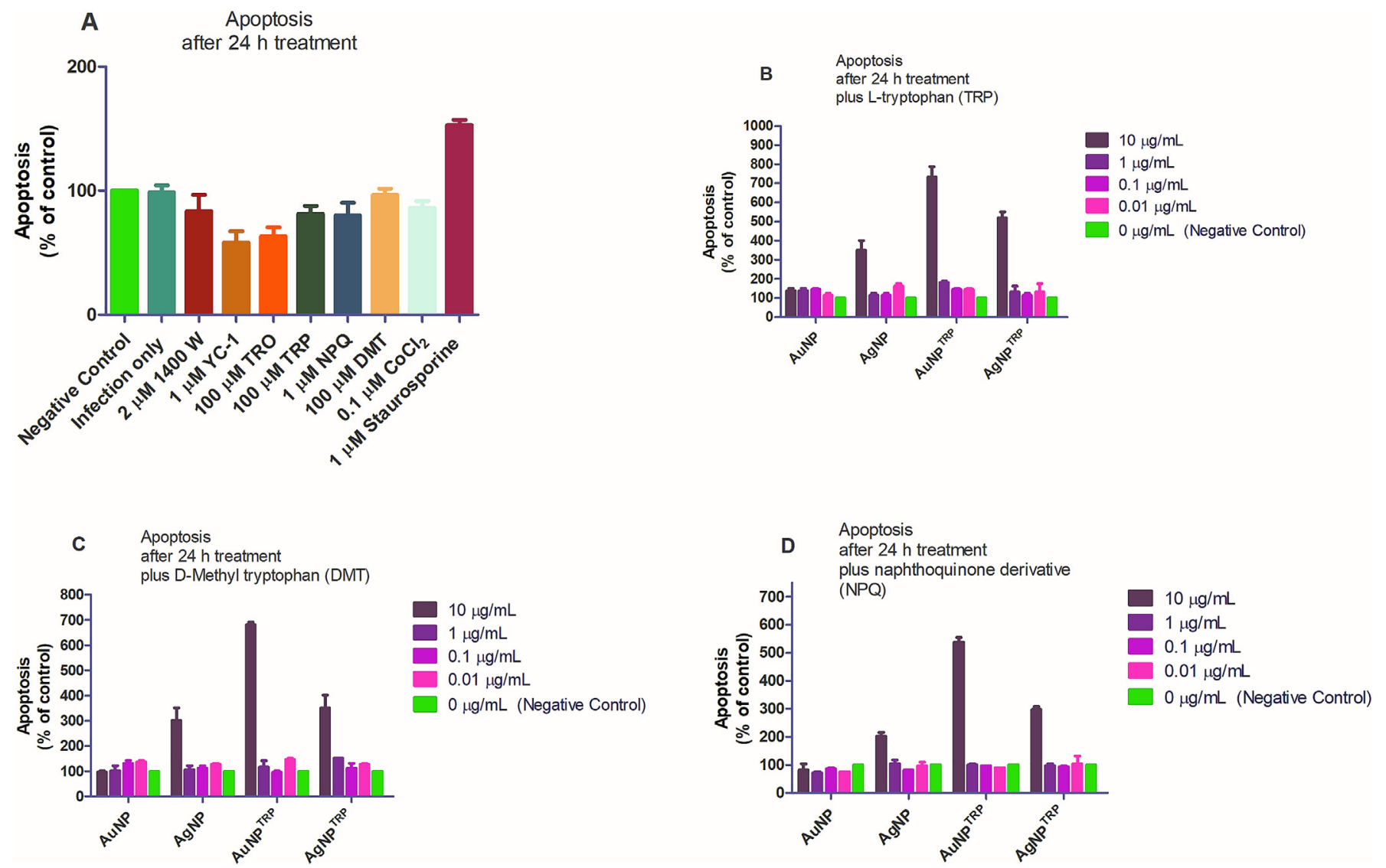

Fig. 4. Cellular apoptosis after 24 treatment; [A] Metabolic modulators only [B] Nanoparticles (NPs) or amino-nanoparticles (amino-NPs) plus L-tryptophan [C] Nanoparticles (NPs) or amino-nanoparticles (amino-NPs) plus 1-Methyl-D-tryptophan (DMT) [D] Nanoparticles (NPs) or amino-nanoparticles (amino-NPs) plus naphthoquinone derivative (NPQ). Data are presented as mean of triplicates \pm standard error of mean (SEM). The experiment was performed in triplicate and repeated three times independently.

cells in 96-well plates with or without $T$. gondii infection were treated with the various nanoparticles (AgNP, AuNP, PtNP, AgNP ${ }^{\mathrm{TRP}}$ and $\mathrm{AuNP}^{\mathrm{TRP}}$ ) alone or in combination with small molecules as metabolic modulators (anti-metabolites). The cells were then incubated for different time points at $37^{\circ} \mathrm{C}$ and $5 \% \mathrm{CO}_{2}$. Thereafter, cellular apoptosis was monitored by colorimetric measurement recorded at $550 \mathrm{~nm}$ on a microplate reader (MTP 500; Corona Electric, Hitachinaka Japan). The biological assay was repeated three times, in triplicate. Selection of doses for the nanoparticles as well as for small molecules was premised on our earlier reports (Adeyemi et al., 2017a, 2017b, 2018b) and on preliminary experiments. In all the cases, doses were less than one third of the $\mathrm{IC}_{50}$ value.

\subsection{Statistical analysis}

Data were analyzed by using a one-way ANOVA (GraphPad Software Inc., San Diego, CA) and are presented as the mean \pm standard error of the mean (SEM). Comparisons among groups were determined by using Dunnett's post-hoc test. $P$-values $<.05$ were considered to be statistically significant.

\section{Results}

AuNP at $5 \mu \mathrm{g} / \mathrm{mL}$ caused cellular apoptosis while AgNP led to a dose dependent apoptosis (Fig. 1a). Likewise, AuNP ${ }^{\text {TRP }}$ caused a dose-dependent apoptosis but $\mathrm{AgNP}^{\mathrm{TRP}}$ only caused cellular apoptosis at the highest dose (Fig. 1b). Meanwhile, addition of trolox appears to mildly mitigate cellular apoptosis by the NPs and amino-NPs (Fig. 1c-d). The cellular apoptosis caused by NPs and amino-NPs do not appear to follow a definite pattern with time kinetics (Fig. 2a-f). Cellular apoptosis by NPs and amino-NPs peaked within $24 \mathrm{~h}$ and further extension of incubation time to 48 and $72 \mathrm{~h}$ showed no appreciable but inconsistent increase in level of apoptosis.

In order to determine whether, cellular apoptosis by NPs and aminoNPs was susceptible to modulation by $T$. gondii infection, we treated infected cells with various concentrations of NPs and amino-NPs and cellular apoptosis was determined after $24 \mathrm{~h}$. T. gondii infection failed to avert cellular apoptosis by NPs and amino-NPs (Fig. 3a-d). Meanwhile, T. gondii infection alone did not cause cellular apoptosis after $24 \mathrm{~h}$.

Furthermore, T. gondii and NPs have been shown to affect similar cellular targets (Adeyemi et al., 2017a, 2017b), therefore, we used small molecules as metabolic modulators (anti-metabolites) of these cellular targets to evaluate how cellular apoptosis by NPs and aminoNPs would be impacted. The various anti-metabolites used in the present study did not cause cellular apoptosis at doses investigated (Fig. 4a). However, in order to validate our apoptosis detection assay, we included staurosporine as a positive drug control. As expected staurosporine caused cellular apoptosis thus validating our detection assay. Of the metabolic modulators investigated in this study, none modulated cellular apoptosis by amino-NPs (Fig. 4b-d and Fig. 5a). But trolox (antioxidant), $\mathrm{CoCl}_{2}$ (chemical inducer of hypoxia) and YC-1 (inhibitor of HIF-1 $\alpha$ ) mildly modulated cellular apoptosis by NPs (Fig. 5b-d). That these metabolic modulators modulate cellular apoptosis by NPs may indicate the relevance of these metabolic processes to the apoptotic action of NPs. 

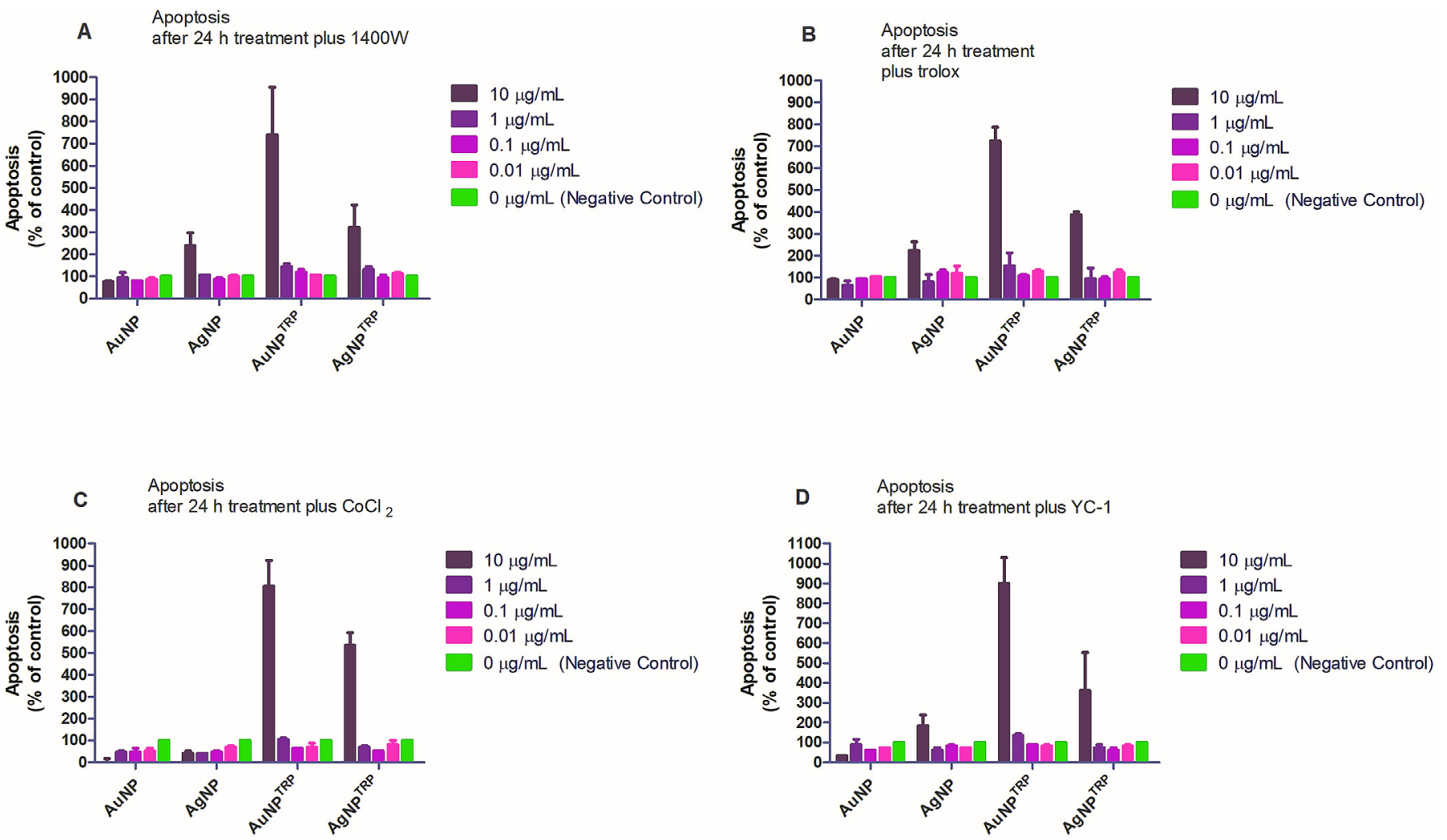

Fig. 5. Cellular apoptosis after 24 treatment with nanoparticles (NPs) or amino-nanoparticles (amino-NPs) plus; [A] $1400 \mathrm{~W}$ (inhibitor of nitric oxide synthase) [B] Trolox [C] $\mathrm{CoCl}_{2}$ (chemical inducer of hypoxia) [D] YC-1 (inhibitor of HIF-1 $\alpha$ protein). Data are presented as mean of triplicates \pm standard error of mean (SEM). The experiment was performed in triplicate and repeated three times independently.

\section{Discussion}

Our current understanding of cellular interactions by NPs is limited. This is because nanomedicine is still an emerging field. Therefore, in this study, we sought to determine the effect of $T$. gondii infection and/ or various anti-metabolites on cellular apoptosis by NPs. The NPs (AgNP) and amino-NPs (AuNP ${ }^{\mathrm{TRP}}$ ) caused cellular apoptosis which appeared to be dose-dependent but do not follow time kinetics. Conversely, cellular apoptosis by AuNP and AgNP ${ }^{\mathrm{TRP}}$ was not dosedependent. Taken together, these findings are consistent with existing findings that have demonstrated the apoptotic action of NPs (Azizi et al., 2017; Moses et al., 2016). Interestingly, the NPs and amino-NPs at the sub-cytotoxicity doses used in this study facilitated cellular apoptosis. This finding confirms earlier report which showed that NPs can increase cellular susceptibility to cell death without necessarily causing cytotoxicity (Leite et al., 2015). The PtNPs exhibited poor cellular apoptosis possibly due to the fact that PtNPs have anti-oxidative stress properties similar to those of cellular antioxidant enzymes (Adeyemi et al., 2018a). Nevertheless, studies have demonstrated in vitro apoptotic tendency of PtNPs in cancer cells, human fibroblast as well as in bacteria cells (Bendale et al., 2017; Kutwin et al., 2017; bacterial cells and HFF cells (Nejdl et al., 2017).

Meanwhile, in the presence of $T$. gondii infection, cellular apoptosis by NPs and amino-NPs was not mitigated. This finding may not only indicate that cellular apoptosis by NPs and amino-NPs was not susceptible to modulation by $T$. gondii infection but that NPs and aminoNPs have antagonistic impact against anti-apoptotic tendency of $T$. gondii. The T. gondii infection has been reported to inhibit pro-apoptotic cellular process, in order to ensure their replication and survival (Hippe et al., 2009). T. gondii does this by affecting the apoptotic cascade at multiple levels; the inhibition of pro-apoptotic effector proteins like Bax and Bak, the avoidance of caspase 9 and Apaf- 1 binding thus preventing caspase $3 / 7$ cascade, upsetting pro- and anti-apoptotic balance of BCL-2 proteins, impeding the function of poly(ADP-ribose) polymerase expression, and upregulating NF-kB pathway by GRA15, which depresses the host immune system through interleukin 12, thereby preventing apoptosis (Hippe et al., 2009; Besteiro, 2015; Kim et al., 2006; BegumHaque et al., 2009; Goebel et al., 2001). Thus, it is plausible that NPs and amino-NPs are antagonist in one or more of these same apoptosis effector sites that are targets of $T$. gondii. This finding supports further the prospects of NPs as alternative anti-parasite therapy.

Small molecules used in this study showed marginal apoptotic features when compared with control as the majority of these anti-metabolites failed to interfere with apoptotic potential of NPs and aminoNPs. For example, trolox mildly modulated the apoptotic potential of NPs. Trolox basically functions by mopping up ROS in the cell, thus our finding confirmed previous studies that showed oxidative stress as one of the cytotoxic mechanisms of NPs (Bagherzadeh Homaee and Ehsanpour, 2016; Mateo et al., 2013). This further suggests the culpability of oxidative stress in the apoptotic action of NPs. That trolox modulated cellular apoptosis by NPs may also indicate that cellular reactivity of these NPs differ from that of amino-NPs. In addition, chemical induction of hypoxia by use of $\mathrm{CoCl}_{2}$ also modulated cellular apoptosis by NPs. It's probable that hypoxia might be interfering with the apoptotic action of NPs. This is further supported by mild mitigation of YC-1 on cellular apoptosis by NPs. More so, NPs have capacity to reduce HIF-1 $\alpha$ activity (Adeyemi et al., 2017b; Yang et al., 2016) and thus avert hypoxia. Therefore, it is plausible that capacity of NPs to downregulate HIF-1 $\alpha$ protein level might be connected to their apoptotic tendency. Taken together, the findings do not only indicate that these metabolic processes (oxidative stress and hypoxia) are relevant to the apoptotic action of NPs but underscore variance in the cellular interactions leading to apoptosis by both NPs and amino-NPs. Data herein support that cellular apoptosis by NPs do not preclude oxidative stress and hypoxia. This is not same for the amino-NPs (AgNP ${ }^{\mathrm{TRP}}$ and $\mathrm{AuNP}^{\mathrm{TRP}}$ ). The reason for this variance behavior in cellular apoptosis 
induction by NPs and amino-NPs is not known yet but may be attributed to change in surface chemistry of amino-NPs occasioned by conjugation of l-tryptophan. This line of thought is consistent with our earlier findings (Adeyemi et al., 2018b) in which NPs-tryptophan conjugates and NPs lacking this amino acid exhibited dissimilar cellular interactions. Furthermore, NPs and amino-NPs have demonstrated capacity to activate kynurenine pathway in vitro (Adeyemi et al., 2017b) by yet unknown mechanism. But in the present study, addition of 1tryptophan to the culture medium failed to ameliorate cellular apoptosis by NPs and amino-NPs. This may indicate that apoptosis by NPs and amino-NPs precludes activation of kynurenine pathway. Moreover, competitive and non-competitive inhibitors of IDO-1 (a rate limiting enzyme in the oxidative degradation of tryptophan to form kynurenine) failed to assuage cellular apoptosis by NPs and amino-NPs.

In conclusion, the present study demonstrates the apoptotic potential of inorganic NPs and amino-NPs of Ag and Au. Further, our findings are evidence supporting that apoptosis by NPs and amino-NPs was not susceptible to modulation by $T$. gondii infection, thus supporting further the prospects of NPs as alternative anti-parasitic agents. Additionally, oxidative stress and chemical hypoxia are partly among the cellular interactions leading to apoptosis caused by NPs. Considered together, the data do not only glean new perspective to deepen our understanding of cellular interactions by NPs and amino-NPs but warrant further investigations in order to fully comprehend mechanistic behavior of NPs.

\section{Acknowledgements}

Authors appreciate JSPS Fellowship to Dr O. S. ADEYEMI and NRCPD Obihiro University of Agriculture \& Veterinary Medicine, Obihiro, Japan.

\section{Conflict of interest}

Authors declare that there is no conflict of interests.

\section{References}

Adeyemi, O.S., Sulaiman, F.A., 2015. Evaluation of metal nanoparticles for drug delivery systems. J. Biomed. Res. 29 (2), 145-149.

Adeyemi, O.S., Murata, Y., Sugi, T., Kato, K., 2017a. Inorganic nanoparticles caused death of Toxoplasma gondii through alteration of redox status and mitochondrial membrane potential. Int. J. Nanomedicine 12, 1647-1661.

Adeyemi, O.S., Murata, Y., Sugi, T., Han, Y., Kato, K., 2017b. Modulation of host HIF-1a activity and the tryptophan pathway contributes to the anti-Toxoplasma gondii potential of nanoparticles. Biochem Biophys Rep. 11, 84-92.

Adeyemi, O.S., Otohinoyi, D.A., Sulaiman, F.A., 2018a. Influence of test model selection on nanotoxicity evaluation. In: Kumar, V., Dasgupta, N., Ranjan, S. (Eds.), Nanotoxicology: Toxicity Evalutaion, Risk Assessment and Management. CRC Press Taylor and Francis, Boca Raton, pp. 144-189.

Adeyemi, O.S., Murata, Y., Sugi, T., Han, Y., Kato, K., 2018b. Exploring amino acidcapped nanoparticles for selective anti-parasitic action and improved host biocompatibility. J. Biomed. Nanotechnol. 14 (5), 847-867 (In Press).

Azizi, M., Ghourchian, H., Yazdian, F., Bagherifam, S., Bekhradnia, S., Nyström, B., 2017 Anti-cancerous effect of albumin coated silver nanoparticles on MDA-MB 231 human breast cancer cell line. Sci. Rep. 7, 5178.
Bagherzadeh Homaee, M., Ehsanpour, A., 2016. Silver nanoparticles and silver ions: Oxidative stress responses and toxicity in potato (Solanum tuberosum L) grown in vitro. Hortic Environ Biote. 57 (6), 544-553.

Begum-Haque, S., Haque, A., Kasper, L., 2009. Apoptosis in Toxoplasma gondii activated T cells: the role of IFN $\gamma$ in enhanced alteration of $\mathrm{Bcl}-2$ expression and mitochondrial membrane potential. Microb. Pathog. 47 (5), 281-288.

Bendale, Y., Bendale, V., Paul, S., 2017. Evaluation of cytotoxic activity of platinum nanoparticles against normal and cancer cells and its anticancer potential through induction of apoptosis. Integrative Medicine Res. 6 (2), 141-148.

Besteiro, S., 2015. Toxoplasma control of host apoptosis: the art of not biting too hard the hands that feeds you. Microb Cell. 2 (6), 178-181.

Blader, I.J., Saeji, J.P., 2009. Communication between Toxoplasma gondii and its host: impact on parasite growth, development, immune evasion and virulence. APMIS 111 (5-6), 458-476.

Goebel, S., Gross, U., Luder, C.G., 2001. Inhibition of host cell apoptosis by Toxoplasma gondii is accompanied by reduced activation of the caspase cascade and alterations of poly(ADP-ribose) polymerase expression. J. Cell Sci. 114 (19), 3495-3505.

Hippe, D., Weber, A., Zhou, L., Chang, D., Hacker, G., Luder, C., 2009. Toxoplasma gondii infection confers resistance against BimS-induced apoptosis by preventing the activation and mitochondrial targeting of pro-apoptotic Bax. J. Cell Sci. 122 (19), 3511-3521.

Kim, J., Ahn, M., Jun, H., Jung, J., Ryu, J., Min, D., 2006. Toxoplasma gondii inhibits apoptosis in infected cells by caspase inactivation and NF-kB activation. Yonsei Med. J. 47 (6), 862-869.

Kutwin, M., Sawosz, E., Jaworski, S., Hinzmann, M., Wierzbicki, M., Hotowy, A., Grodzik, M., Winnicka, A., Chwaliboq, A., 2017. Investigation of platinum nanoparticle properties against U87 glioblastoma multiforme. Arch. Med. Sci. 6, 1322-1334.

Leite, P.E., Pereira, M.R., do Nascimento Santos, C.A., Campos, A.P., Esteves, T.M., Granjeiro, J.M., 2015. Gold nanoparticles do not induce myotube cytotoxicity but increase the susceptibility to cell death. Toxicol. in Vitro 29 (5), 819-827.

Lüder, C.G.K., Gross, U., 2005. Apoptosis and its Modulation during infection with Toxoplasma gondii: molecular mechanisms and role in pathogenesis. In: Griffin, D.E. (Ed.), Role of Apoptosis in Infection. Current Topics in Microbiology and Immunology. 289. Springer, Berlin, Heidelberg, pp. 219-237.

Mateo, D., Morales, P., Ávalos, A., Haza, A., 2013. Oxidative stress contributes to gold nanoparticle-induced cytotoxicity in human tumor cells. Toxicol Mech Meth. 24 (3), 161-172.

Moses, S.L., Edwards, V.M., Brantley, E., 2016. Cytotoxicity in MCF-7 and MDA-MB-231 breast cancer cells, without harming MCF-10A healthy cells. J Nanomed Nanotechnol. 7, 369.

Nejdl, L., Kudr, J., Moulick, A., Hegerova, D., Ruttkay-Nedecky, B., Gumulec, J., Cihalova, K., Snerkova, K., Dostava, S., Kriskova, S., Novotna, M., Kopel, P., Adma, V., 2017. Platinum nanoparticles induce damage to DNA and inhibit DNA replication. PLoS One 12 (7), e0180798.

Rieznichenko, L.S., Dybkova, S.M., Gruzina, T.G., Ulberg, Z.R., Todor, I.N., Lukyanova, N.Y., Shpyleva, S.I., Chekhun, V.F., 2012. Gold nanoparticles synthesis and biological activity estimation in vitro and in vivo. Exp. Oncol. 34 (1), 25-28.

Satapathy, S.R., Mohapatra, P., Preet, R., Das, D., Sarkar, B., Choudhuri, T., Wyatt, M.D. Kundu, C.N., 2013. Silver-based nanoparticles induce apoptosis in human colon cancer cells mediated through p53. Nanomedicine 8 (8), 1307-1322.

Selim, M.E., Hendi, A.A., 2012. Gold nanoparticles induce apoptosis in MCF-7 human breast cancer cells. Asian Pac. J. Cancer Prev. 13 (4), 1617-1620.

Spear, W., Chan, D., Coppens, I., Johnson, R.S., Giaccia, A., Blader, I.J., 2006. The host cell transcription factor hypoxia-inducible factor 1 is required for Toxoplasma gondii growth and survival at physiological oxygen levels. Cell. Microbiol. 8, 339-352.

Sulaiman, F., Akanji, M., Oloyede, H., Sulaiman, A., Olatunde, A., Joel, E., Adewale, T.H., Adeboye, H.A., Idris, S.O., Quadri, A.L., Oyegoke, O.S., Adeyemi, O.S., 2015. Oral exposure to silver/gold nanoparticles: status of rat lipid profile, serum metabolites and tissue morphology. J Med Sci (Faisalabad). 15 (2), 71-79.

Wiley, M., Sweeney, K.R., Chan, D.A., Brown, K.M., McMurtrey, C., Howard, E.W., Giaccia Aj Blader, I.J., 2010. Toxoplasma gondii activates hypoxia-inducible factor (HIF) by stabilizing the HIF-1alpha subunit via type I activin-like receptor kinase receptor signaling. J. Biol. Chem. 285 (35), 26852-26860.

Yang, T., Cao, F., Liu, Q., Wang, X., 2016. Silver nanoparticles inhibit hypoxia inducible factor function and cancer cell growth. Nanomedicine 12 (2), 554.

Zinkernagel, A.S., Johnson, R.S., Nizet, V., 2007. Hypoxia-inducible factor (HIF) function in innate immunity and infection. J. Mol. Med. 85, 1339-1346. 